TRANSACTIONS OF THE

AMERICAN MATHEMATICAL SOCIETY

Volume 355, Number 2, Pages 519-538

S 0002-9947(02)03094-

Article electronically published on October 1, 2002

\title{
THE OREVKOV INVARIANT OF AN AFFINE PLANE CURVE
}

\author{
WALTER D. NEUMANN AND PAUL NORBURY
}

\begin{abstract}
We show that although the fundamental group of the complement of an algebraic affine plane curve is not easy to compute, it possesses a more accessible quotient, which we call the Orevkov invariant.
\end{abstract}

\section{INTRODUCTION}

An interesting topological invariant of an algebraic affine plane curve $\Sigma \subset \mathbb{C}^{2}$ is its link at infinity $L_{\Sigma}$ obtained by intersecting $\Sigma$ with a large sphere in $\mathbb{C}^{2}$. It is quite easily computed using 9, 10 .

From $L_{\Sigma}$ and the number of reducible components of $\Sigma$, one can retrieve the arithmetic genus of $\Sigma$ 9, 10, and much about the topology of the defining polynomial $f$ given by $\Sigma=\{f=0\}$, including the topology of its generic fibre and information about its local and global monodromy representations [4, 11]. The link $L_{\Sigma}$ also enables one to obtain geometric information about the curve beyond homological information [12]. This raises the question: how much can $L_{\Sigma}$ tell us about $\pi_{1}\left(\mathbb{C}^{2}-\Sigma\right)$ ?

If $\Sigma$ is a generic fibre of its defining polynomial $f$, then $L_{\Sigma}$ gives the topology of $\Sigma$ as an embedded curve in $\mathbb{C}^{2}$, and hence it determines $\pi_{1}\left(\mathbb{C}^{2}-\Sigma\right)$. (In fact, $\pi_{1}\left(\mathbb{C}^{2}-\Sigma\right)=\mathbb{Z}$ in this case by Oka 15 . See Section 2, Theorem 2.2.) In contrast, the cuspidal and nodal curves $x^{2}=y^{3}$ and $x^{2}=y^{3}+1$ both have the trefoil at infinity, whereas the fundamental groups of their complements are respectively the braid group $\{a, b \mid a b a=b a b\}$ and $\mathbb{Z}$. This leads us to consider a common quotient of the different fundamental groups.

Definition 1. If $p$ is a singular point of a plane curve $\Sigma$, let $G_{p}=\pi_{1}\left(B_{p} \cap\left(\mathbb{C}^{2}-\Sigma\right)\right)$ for a sufficiently small ball $B_{p}$ around $p$. The Orevkov invariant of $\Sigma$ is

$$
\mathcal{O}(\Sigma)=G / N
$$

where $G=\pi_{1}\left(\mathbb{C}^{2}-\Sigma\right)$ and $N$ is the normal closure in $G$ of the union over all singular points $p$ of $\Sigma$ of the images of the commutator subgroups $\left[G_{p}, G_{p}\right]$.

Remark. The image of $G_{p}$ in $G$ is only well-defined up to conjugation, but since we take the normal closure, $N$ is well-defined.

When the invariant $\mathcal{O}(\Sigma)$ is abelian, it is $H_{1}\left(\mathbb{C}^{2}-\Sigma\right)=\mathbb{Z}^{k}$, where $k$ is the number of irreducible components of $\Sigma$. This invariant was first studied by Orevkov in [17]; he proved that it is abelian when $L_{\Sigma}$ is a positive braid. (He also showed that

Received by the editors November 17, 2001.

2000 Mathematics Subject Classification. Primary 14H30, 14R10, 57M25.

Supported under NSF grant no. DMS-0083097. 
the invariant is abelian on projective plane curves, which generalises the (solved) Zariski conjecture [16].) The invariant ignores the complications of configurations of hyperplanes, since it is abelian in these cases.

In this paper, we will show that the Orevkov invariant of an algebraic affine plane curve is invariant under a special class of deformations of the curve. By "deformation" we would normally mean a complex analytic family, but the deformations we consider need only be complex analytic near the singularities.

Definition 2. A deformation of a singularity germ is local component preserving (LCP) if it does not change the number of local irreducible components near the singularity. An LCP deformation of a curve is a deformation that is an LCP deformation in a neighbourhood of each singularity, and is a smooth proper isotopy outside smaller neighbourhoods of the singularities.

This definition applies to projective or affine curves. For affine curves the condition that the isotopy is proper means that the link at infinity is preserved. For example, if $\Sigma$ is the image of a non-constant analytic map $f_{0}: \mathbb{C} \rightarrow \mathbb{C}^{2}$, then any complex analytic deformation $f_{t}$ of $f_{0}$ that preserves the link at infinity gives an LCP deformation of $\Sigma$. Examples of non-LCP deformations are:

(1) the deformation $\Sigma_{\epsilon}=\{(x, y): x y=\epsilon\}$ of $\Sigma_{0}=\{(x, y): x y=0\}$ (not LCP at the singularity);

(2) the deformation $\Sigma_{\epsilon}=\left\{(x, y): x y^{2}+y=\epsilon\right\}$ of $\Sigma_{0}=\left\{(x, y): x y^{2}+y=0\right\}$ (not proper; link at infinity is not preserved).

Theorem 1. The Orevkov invariant is invariant under LCP deformations.

A polynomially parametrised curve is a curve with irreducible components given by algebraic maps from $\mathbb{C}$ to $\mathbb{C}^{2}$. Each irreducible component can be parametrised by a pair of single-variable polynomials and is thus a rational curve with one place at infinity.

Polynomially parametrised curves arise naturally as the set of non-generic regular values of a self-map of $\mathbb{C}^{2}$ as follows. Consider a polynomial map $F: \mathbb{C}^{2} \rightarrow \mathbb{C}^{2}$ with two-dimensional image. The pre-image of a generic value consists of $d$ points, say. There are two classes of non-generic values: those that are regular and those that are irregular. The pre-image of a non-generic regular value consists of fewer than $d$ points. The closure (in $\mathbb{C}^{2}$ ) of the set of non-generic regular values is a polynomially parametrised curve. This is because the missing points in the preimage lie "at infinity". If we compactify $\mathbb{C}^{2}$ by including a divisor at infinity so that $\bar{F}$ is well-defined there, then the points of the divisor at infinity that map to finite values under $\bar{F}$ consist of some of the rational curves of the divisor at infinity. These rational curves are disjoint and each rational curve contains exactly one point that is mapped to infinity, and hence the image of the union of the rational curves is a polynomially parametrised curve. The image of the rational curves at infinity might intersect the set of irregular values, and hence it gives the closure (in $\mathbb{C}^{2}$ ) of the non-generic regular points.

If one restricts to deformations of a polynomially parametrised curve that remain within the class of polynomially parametrised curves, then automatically the LCP condition is satisfied at singularities. Hence, from Theorem[1, we see that in many cases the Orevkov invariant of a polynomially parametrised curve is related to its link at infinity. 
Corollary 1.1. The Orevkov invariant of a polynomially parametrised curve depends only on its link at infinity when the moduli space of polynomially parametrised curves with that link at infinity is connected.

Let $X_{\Sigma}$ be a compactification of $\mathbb{C}^{2}$ on which $\Sigma$ meets the divisor at infinity $D$ transversally. The link at infinity $L_{\Sigma}$ encodes the minimal such divisor $D$ (see, e.g., [10]) and it also encodes the canonical class of $X_{\Sigma}$ supported on $D$. Each component of the link corresponds to a rational curve in $D$, known as a horizontal curve. When the canonical class at each link component is negative enough on each horizontal curve, we can prove that the moduli space of polynomially parametrised curves with link at infinity $L_{\Sigma}$ is connected and use this to prove the following theorem.

Theorem 2. For sufficiently negative canonical class on each horizontal curve of $X_{\Sigma}$, the Orevkov invariant $\mathcal{O}(\Sigma)$ of a polynomially parametrised curve $\Sigma$ is abelian.

We state precisely how negative is sufficient in Section 5.2, Theorem 5.2, and in Section 5.3 we show that the class of positive braids $L_{\Sigma}$ is strictly contained in this set. Note that Orevkov [17] proved that the Orevkov invariant is abelian on any curve with positive braid at infinity, not just polynomially parametrised curves with such a property. Since our sufficiently negative condition is only a mild improvement of Orevkov's result in the polynomially parametrised case, it is the connectivity of certain moduli spaces proved in this paper that is the more significant improvement of his result.

An example of a link at infinity that satisfies the conditions of Theorem 2 is the $(5,2)$ cabling on the $(2,3)$ torus knot, which is not a positive braid, and is represented by the following splice diagram:

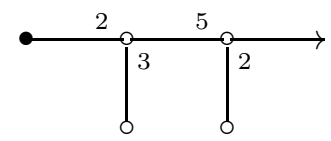

\section{NODAl CURVES}

When the curve $\Sigma$ is a nodal curve - its only singularities are simple double points - then $\mathcal{O}(\Sigma)=\pi_{1}\left(\mathbb{C}^{2}-\Sigma\right)$. The fundamental group of the complement of a nodal plane curve has a long history, beginning with the Zariski conjecture, proven by Deligne [3] and Fulton [ [6], that the complement of a projective plane nodal curve has abelian fundamental group. This was generalised by Nori 14] to projective and affine curves in smooth surfaces.

Theorem 2.1 (Nori). Let $\Sigma$ and $D$ be curves on a smooth projective surface $X$, that intersect transversally. Assume that $\Sigma$ is nodal. Denote the number of singular points on a curve $C$ by $r(C)$. Assume that $C \cdot C>2 r(C)$ for every irreducible component $C$ of $\Sigma$. Then, if $N$ denotes the kernel of $\pi_{1}(X-\Sigma \cup D) \rightarrow \pi_{1}(X-D)$, $N$ is a finitely generated abelian group and the centraliser of $N$ is a subgroup of finite index.

In particular, when $\Sigma$ is a nodal curve in $\mathbb{C}^{2}$, and $X$ the blow-up of $\mathbb{P}^{2}$ that resolves the singularities of $\Sigma$ at infinity, and $D=X-\mathbb{C}^{2}$ the divisor at infinity, if $\bar{\Sigma}_{i} \cdot \bar{\Sigma}_{i}>2 r\left(\Sigma_{i}\right)$ for each irreducible component $\Sigma_{i}$ of $\Sigma$, then $\pi_{1}\left(\mathbb{C}^{2}-\Sigma\right)$ is abelian (since $X-D=\mathbb{C}^{2}$ and $N=\pi_{1}\left(\mathbb{C}^{2}-\Sigma\right)$ in the theorem). 
As mentioned in the introduction, when $\Sigma$ is the generic fibre of its defining polynomial, $L_{\Sigma}$ determines $\pi_{1}\left(\mathbb{C}^{2}-\Sigma\right)$.

Theorem 2.2 (Oka [15]). If $\Sigma$ is the generic fibre of a polynomial $f$, then

$$
\pi_{1}\left(\mathbb{C}^{2}-\Sigma\right)=\mathbb{Z}
$$

Proof. Choose a disk $D_{s} \subset \mathbb{C}$ to contain all of the atypical values of $f$ and a small disjoint disk $D_{c}$ that gives a neighbourhood of $c=f(\Sigma)$. Join the disks by a path $\gamma$ and put $D^{\prime}=D_{s} \cup \gamma \cup D_{c}$. Then each of the following arrows is a homotopy equivalence:

$$
\begin{gathered}
f^{-1}\left(D_{s}\right) \hookrightarrow f^{-1}\left(D_{s} \cup \gamma\right) \hookrightarrow f^{-1}\left(D^{\prime}\right) \hookrightarrow \mathbb{C}^{2}, \\
f^{-1}\left(D^{\prime}-\{c\}\right) \hookrightarrow \mathbb{C}^{2}-\Sigma .
\end{gathered}
$$

Apply the Seifert Van-Kampen theorem to

$$
f^{-1}\left(D^{\prime}-\{c\}\right)=f^{-1}\left(D_{s} \cup \gamma\right) \cup f^{-1}\left(D_{c}-\{c\}\right) .
$$

Since $f^{-1}\left(D_{c}-\{c\}\right) \cong\left(D_{c}-\{c\}\right) \times \Sigma \sim S^{1} \times \Sigma$, we get that $\pi_{1}\left(\mathbb{C}^{2}-\Sigma\right)$ is the quotient of $\pi_{1}\left(\mathbb{C}^{2}\right) * \pi_{1}\left(S^{1} \times \Sigma\right)=\pi_{1}\left(S^{1} \times \Sigma\right)$ by the normal closure of $\pi_{1}(\Sigma)$, and thus $\pi_{1}\left(\mathbb{C}^{2}-\Sigma\right)=\mathbb{Z}$.

Remark. It is amusing to note that Theorem 2.2 is almost a consequence of Theorem 2.1, which requires $\bar{\Sigma} \cdot \bar{\Sigma}>0=2 r(\Sigma)$ to conclude that $\pi_{1}\left(\mathbb{C}^{2}-\Sigma\right)$ is abelian. The self-intersection number of the generic fibre of a polynomial is nonnegative. It is given as a sum over linking numbers of components of $L_{\Sigma}$. In [10] it was shown that the polynomial is irregular at a point at infinity precisely when the linking number for the corresponding link component is 0 . In particular, for a good polynomial, one which is never irregular at infinity, the self-intersection number of the generic fibre is strictly positive and Theorem 2.1 implies Theorem 2.2 In fact, Theorem 2.1 applies in most cases, since it is quite rare that the generic fibre of a polynomial has zero self-intersection or, equivalently, that it is irregular at infinity at each link component of $L_{\Sigma}$. Russell's bad field generator [18] and each polynomial in Kaliman's classification of rational polynomials with a $\mathbb{C}^{*}$ fibre [7] are examples of polynomials whose generic fibre has zero self-intersection number, and hence Theorem 2.1 does not imply Theorem 2.2 in these cases.

This paper will consist mainly of examples of curves with abelian Orevkov invariant. Two examples with nonabelian invariant are as follows.

(i) For $f: \mathbb{C}^{2} \rightarrow \mathbb{C}$ the curve $\Sigma=f^{-1}(A)$ for a finite set of at least two points $A \subset \mathbb{C}$ has nonabelian Orevkov invariant.

(ii) The following example was constructed by Mutsuo Oka. Let

$$
f(x, y)=\left(x^{2}-y\right)^{2} y-4 x\left(x^{2}-y\right)+4=0 .
$$

Then $\Sigma=f^{-1}(0)$ is a smooth irreducible curve with

$$
\pi_{1}\left(\mathbb{C}^{2}-\Sigma\right)=\{a, b \mid a b a=b a b\}=\mathcal{O}(\Sigma) .
$$

\section{INVARIANCE UNDER LOCAL DEFORMATIONS}

In this section we will prove Theorem 11 by showing that it reduces easily to an argument of Orevkov's. 
Proof. Given a plane curve $\Sigma$, we can enclose its singularities in small balls $B_{i}$ and compute $\pi_{1}\left(\mathbb{C}^{2}-\Sigma\right)$ by the Van Kampen theorem in terms of the fundamental groups of the complement of $\Sigma$ in each of the balls as well as their exterior $X=$ $\mathbb{C}^{2}-\bigcup_{i} B_{i}$. Under an LCP deformation, we can assume that the topology of $X-(\Sigma \cap X)$ does not change; so it suffices to show that the Orevkov invariant of each $\left(B_{i}, B_{i} \cap \Sigma\right)$ also does not change.

We therefore restrict to the local situation, in which we have a germ of a singularity $(\Sigma, p)$ and a small ball $B$ around $p$ such that its boundary and the boundary of all smaller balls centered at $p$ intersect $\Sigma$ transversally. We take an LCP deformation that changes the intersection of $\Sigma$ with $\partial B$ only by an isotopy. In this situation, we must show that the Orevkov invariant of $(B, B \cap \Sigma)$ does not change. We may change coordinates so that $p$ is the origin and $\Sigma$ is given by an equation $f(x, y)=0$ of degree $n$ with $f(x, y)=x^{n}+a_{n-1}(y) x^{n-1}+\cdots+a_{0}(y)$. We may then take the ball $B$ to be the polydisk $\left\{(x, y):|x| \leq \epsilon_{1},|y| \leq \epsilon_{2}\right\}=D_{1} \times D_{2}$, chosen such that $\Sigma$ intersects $\partial B$ only in the portion $\partial D_{1} \times D_{2}$. Finally, we may assume our coordinates are chosen so that the set of $x$ for which $\Sigma \cap B$ contains more than one point $(x, y)$ whose $y$-coordinates have the same real part is in generic position in the sense of Orevkov's papers 16] and 17, both before and after our deformation. We denote this set

$$
P:=\left\{x \in D_{1}: \exists y_{1} \neq y_{2} \text { with }\left(x, y_{1}\right) \in \Sigma,\left(x, y_{2}\right) \in \Sigma, \Re y_{1}=\Re y_{2}\right\} .
$$

Generic position implies that $P$ consists of a collection of paths oriented by increasing $\left|y_{1}-y_{2}\right|$. Before deforming $\Sigma$, these paths start at the image of the one singular point and fan out to $\partial D_{1}$, since we choose the ball small enough that the only singular point of the projection $B \cap \Sigma \rightarrow D_{1}$ is the singular point of $\Sigma$. After the deformation, there may be several singular points, but we may assume that the behavior of the paths near $\partial D_{1}$ has changed only by an isotopy. The paths then still start at singular values of the projection $B \cap \Sigma \rightarrow D_{1}$ and end at the boundary of the disk. Moreover, away from singular points of the projection $B \cap \Sigma \rightarrow D_{1}$, the paths may now intersect, but Orevkov's genericity condition says that they intersect at most in pairs or in triples, and these intersections are transverse (the triple intersections arise when three real parts of $y$-values coincide at a time). The projection $B \cap \Sigma \rightarrow D_{1}$ is a covering map on the inverse image of $D_{1}-P$, of degree $d$, say, and, as in [16], one can give a presentation of $\pi_{1}(B-\Sigma)$ with $d$ generators for each component of $D_{1}-P$, and with relations associated to the singular points of $B \cap \Sigma \rightarrow D_{1}$ and to the intersection points of paths comprising $P$. The same inductive argument that Orevkov uses, moving along the paths starting from the singular points, then shows that the Orevkov invariant is free abelian with one generator for each irreducible component of $B \cap \Sigma$.

\section{Splice Diagrams}

A convenient way to represent the link at infinity of an affine plane algebraic curve uses the splice diagram of the link. The splice diagram of a link at infinity can be viewed as a cabled torus knot in $S^{3}$, or the Puiseux expansion of the affine curve at infinity given by expressing one coordinate in terms of the other, or the plumbing graph of an efficient resolution at infinity of the affine curve. We will demonstrate these three views by focusing on the example of a curve with knot at infinity. This includes irreducible polynomial curves. The splice diagram of such a 
curve is given by where the pairs of integers $\left(p_{k}, q_{k}\right)$ are known as weights of the

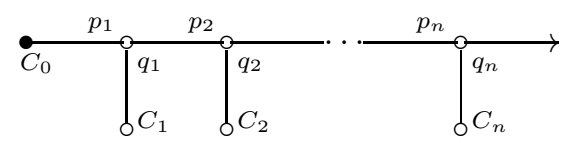

FiguRE 1. Splice diagram at infinity

splice diagram and the $C_{k}$ are simply labels for the valency-one nodes.

The splice diagram represents a knot obtained by cabling a $\left(p_{2}, q_{2}\right)$ torus knot on a $\left(p_{1}, q_{1}\right)$ torus knot and then cabling a $\left(p_{3}, q_{3}\right)$ torus knot on that, and so on. The earlier torus knots are represented as "virtual" link components in the diagram by the valency-one nodes $C_{k}$. For example, the components $C_{0}$ and $C_{1}$ form a Hopf link, and the component $C_{2}$ is a $\left(p_{1}, q_{1}\right)$ torus knot cabled on $C_{1}$. The arrowhead represents the actual knot, a $\left(p_{n}, q_{n}\right)$ cabling on the virtual knot $C_{n}$.

The linking number of any two (virtual) components can be calculated from the splice diagram. It is the product of all weights incident to a path joining the two nodes. Thus, $l\left(C_{j}, C_{k}\right)=p_{j} q_{j+1} \ldots q_{k}$, and if we denote the knot (represented by the arrowhead) by $K$, then $l\left(C_{k}, K\right)=p_{k} q_{k+1} \ldots q_{n}$.

Not all knots arise as the knot at infinity of a plane curve. Necessary and sufficent conditions on the weights of a splice diagram of a knot at infinity are:

$$
\left\{\begin{array}{l}
\text { i. } p_{k}>1, q_{k}>1,\left(p_{k}, q_{k}\right)=1 ; \\
\text { ii. } \Delta_{k}=p_{k}-p_{k-1} q_{k-1} q_{k}<0 \text { and } \Delta_{1}=p_{k}-q_{k}<0 ; \\
\text { iii. } p_{k+1} \in \mathbb{N} q_{1} q_{2} \ldots q_{k} \oplus \mathbb{N} p_{1} q_{2} \ldots q_{k} \oplus \cdots \oplus \mathbb{N} p_{k-1} q_{k} \oplus \mathbb{N} p_{k} .
\end{array}\right.
$$

The third, rather deep, property, involving linking numbers of virtual link components, is the semi-group condition of Abhyankar and Moh [1].

A plane curve $\Sigma$ that has this knot at infinity is a degree $q_{1} \ldots q_{n}$ curve and furthermore, we can choose coordinates $x$ and $y$ so that the defining polynomial $P(x, y)$ of $\Sigma$ has a single highest degree term $y^{q_{1} q_{2} \ldots q_{n}}$ and the highest degree monomial in $x$ is $x^{p_{1} q_{2} \ldots q_{n}}$. Then one can expand $y$ in terms of $x$ :

(2) $y=x^{p_{1} / q_{1}}\left(a_{10}+a_{11} x^{e_{11} / q_{1}}+a_{12} x^{e_{12} / q_{1}}+\ldots+x^{e_{2} / q_{1} q_{2}}\left(a_{20}+a_{21} x^{e_{21} / q_{1} q_{2}}+\ldots\right.\right.$

for decreasing exponents $0>e_{11} / q_{1}>e_{12} / q_{1}>\ldots>e_{2} / q_{1} q_{2}>\left(e_{2}+e_{21}\right) / q_{1} q_{2}>\ldots$ where $e_{k}=\Delta_{k}$ and the highest degree term in which the denominator of the exponent does not divide $q_{1} \ldots q_{k-1}$ is $a_{k 0} x^{e_{k} / q_{1} q_{2} \ldots q_{k}}\left(a_{k 0} \neq 0,\left(e_{k}, q_{k}\right)=1\right)$. We can express $y$ more neatly as

$y=x^{p_{1} / q_{1}}\left(r_{1}\left(x^{-1 / q_{1}}\right)+x^{\Delta_{2} / q_{1} q_{2}}\left(r_{2}\left(x^{-1 / q_{1} q_{2}}\right)+\ldots+x^{\Delta_{k} / q_{1} \ldots q_{k}}\left(r_{k}\left(x^{-1 / q_{1} \ldots q_{k}}\right)+\ldots\right.\right.\right.$ for polynomials $r_{k}$ with $r_{k}(0) \neq 0$ and degree $r_{k}<-\Delta_{k+1} / q_{k+1}$. Alternatively, in keeping with the approaches of [5, 10], where the expansion at infinity is obtained by compactifying and taking a local expansion around the point at infinity, we can make the expansion homogeneous by introducing the coordinate at infinity $z$. The expansion becomes

$y=\left(x^{-1} z\right)^{p_{1} / q_{1}} z\left(r_{1}\left(\left(x^{-1} z\right)^{1 / q_{1}}\right)+\ldots+\left(x^{-1} z\right)^{-\Delta_{k} / q_{1} \ldots q_{k}}\left(r_{k}\left(\left(x^{-1} z\right)^{1 / q_{1} \ldots q_{k}}\right)+/ \ldots\right.\right.$, and, setting $x=1$, we get an expansion for $y$ in terms of $z$ :

$y=z^{1-p_{1} / q_{1}}\left(r_{1}\left(z^{1 / q_{1}}\right)+z^{-\Delta_{2} / q_{1} q_{2}}\left(r_{2}\left(z^{1 / q_{1} q_{2}}\right)+\ldots+z^{-\Delta_{k} / q_{1} \ldots q_{k}}\left(r_{k}\left(z^{1 / q_{1} \ldots q_{k}}\right)+\ldots\right.\right.\right.$, 
which gives the splice diagram

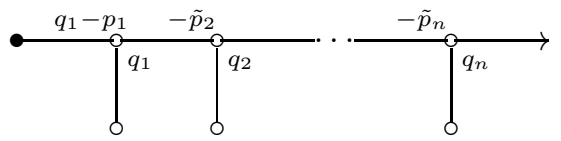

for $\tilde{p}_{k}=p_{k}+\xi_{k}$, where $\xi_{k}$ is chosen so that the edge determinant is $-\Delta_{k}$. By [9], this local splice diagram converts to the splice diagram at infinity given in (A).

The splice diagram also gives an efficient resolution around the point of $\Sigma$ at infinity. Compactify $\mathbb{C}^{2}$ to $\mathbb{P}^{2}$ and let $\mathcal{P}(y, z)=0$ define $\bar{\Sigma}$ in a neighbourhood of the point $[1: 0: 0] \in \mathbb{P}^{2}$ (which we have supposed to be the point where $\bar{\Sigma}$ meets the line at infinity). Each characteristic pair in the splice diagram encodes a multiple blow-up of the resolution of the point at infinity. To resolve at the $k$ th step, one replaces $(y, z)$ by $\left(y^{a} z^{-\Delta_{k}}+Q_{k}\left(y^{b} z^{q_{k}}\right), y^{b} z^{q_{k}}\right)$, where $a, b>0$ are chosen so that $a q_{k}+b \Delta_{k}=1$. The single-variable polynomial $Q_{k}$ is uniquely determined. The curve $\bar{\Sigma}$ is "resolved" at infinity when it meets the divisor at infinity transversally. Each node $C_{k}$ represents a curve in the divisor at infinity, and the defining polynomial of the curve $\bar{\Sigma}$ in a neighbourhood of $C_{k}$ is the polynomial obtained at the $k$ th step of the resolution.

The approximate roots of the defining polynomial $P(x, y)$ of $\Sigma$,

$$
P_{2}=P^{1 / q_{2} \ldots q_{n}}, \ldots, P_{k}=P^{1 / q_{k} \ldots q_{n}}, \ldots, P_{n}=P^{1 / q_{n}},
$$

are each defined uniquely by the respective condition

$$
\operatorname{deg}_{y}\left(P-P_{k}^{q_{k} \ldots q_{n}}\right)<q_{1} \ldots q_{n}-q_{1} \ldots q_{k-1} .
$$

It is easy to calculate each of these by setting

$$
P_{k}=y^{q_{1} \ldots q_{k-1}}+b_{1}(x) y^{q_{1} \ldots q_{k-1}-1}+\cdots+b_{q_{1} \ldots q_{k-1}}(x)
$$

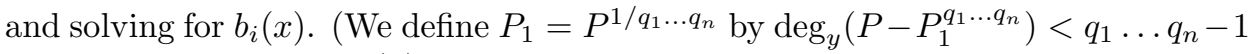
and a priori $P_{1}=y-b(x)$, although by choice of coordinates, $P_{1}=y-y_{0}$ for a constant $y_{0}$.)

The zero set of the polynomial $P_{k}$ defines a curve that meets the splice diagram or, equivalently, the divisor at infinity, at $C_{k}$. This follows from the fact that when we resolve $P$, to get $\tilde{P}$, the approximate $1 / q_{k} \ldots q_{n}$ root $P_{k}$ resolves to give the approximate $1 / q_{k} \ldots q_{n}$ root of $\tilde{P}$. Furthermore, restricted to $C_{k}, \tilde{P}$ is a degree $q_{k} \ldots q_{n}$ polynomial with approximate $1 / q_{k} \ldots q_{n}$ root given by the resolution of $P_{k}$; so the latter must be a coordinate.

Consider all holomorphic functions on $\Sigma$, meromorphic at infinity. The orders of the poles of these functions form a semi-group in $\mathbb{N}$ known as the Weierstrass semi-group, with complement the Weierstrass gap sequence. Using Riemann-Roch, the size of the Weierstrass gap sequence can be shown to be equal to the arithmetic genus $g$ of $\Sigma$, which is easily calculated in terms of the splice diagram:

$$
1-2 g=q_{1} \ldots q_{n}+\sum_{i=1}^{n-1} p_{i}\left(1-q_{i}\right) q_{i+1} \ldots q_{n}+p_{n}\left(1-q_{n}\right) .
$$

Theorem 4.1. The Weierstrass semi-group is generated by the orders of the poles of the holomorhic functions on $\Sigma$ given by the coordinates $x$ and $y$ and the approximate roots $P_{2}, \ldots, P_{n}$. 
Proof. The semi-group $H_{n}$ generated by the poles of $x$ and $y$ and the approximate roots $P_{2}, \ldots, P_{n}$ at $\infty \in \bar{\Sigma}$ is contained in the Weierstrass semi-group; so it is sufficient to show that the size of the gap $G_{n}=\mathbb{N}-H_{n}$ is equal to the size of the Weierstrass gap sequence.

The order of the pole of the approximate root $P_{k}$ can be calculated from the link at infinity. It is given by the linking number of the knot at infinity of $\Sigma$ with the knot at infinity of $P_{k}$, and the latter is the virtual knot represented by the node $C_{k}$ of the splice diagram. Thus the order of the pole of $P_{k}$ is $l\left(C_{k}, K\right)=p_{k} q_{k+1} \ldots q_{n}$, and $l\left(C_{n}, K\right)=p_{n}, l\left(C_{0}, K\right)=q_{1} q_{2} \ldots q_{n}, l\left(C_{1}, K\right)=p_{1} q_{2} \ldots q_{n}$, are the respective orders of the poles of $P_{n}, x$ and $y$. For $k=1, \ldots, n$, put

$$
H_{k}=\mathbb{N} q_{1} q_{2} \ldots q_{k} \oplus \mathbb{N} p_{1} q_{2} \ldots q_{k} \oplus \mathbb{N} p_{2} q_{3} \ldots q_{k} \oplus \cdots \oplus \mathbb{N} p_{k-1} q_{k} \oplus \mathbb{N} p_{k}
$$

so the semi-group condition of Abhyankar and Moh can be restated as $p_{k} \in H_{k-1}$.

Any element $a \in H_{k}$ has a unique normal form given by

$$
a=\sum_{i=0}^{n} a_{k} \cdot l\left(C_{i}, C_{k}\right)=a_{0} \cdot q_{1} q_{2} \ldots q_{k}+\sum_{i=1}^{k-1} a_{i} \cdot p_{i} q_{i+1} \ldots q_{k}+a_{k} \cdot p_{k}
$$

for nonnegative integers $a_{i}$ satisfying $a_{i}<q_{i}(i=1, \ldots, k)$ and $a_{0}$ unrestricted. This follows easily from the fact that since $p_{k} q_{k} \in H_{k-1} q_{k} \subset H_{k}$, in any expression for $a$ we can reduce the coefficient of $p_{k}$ by multiples of $q_{k}$, redistributing the quantity amongst the previous coefficients of $l\left(C_{i}, C_{k}\right)$ for $i<k$. A similar step can then be taken to adjust the coefficient of $p_{k-1} q_{k}$ by multiples of $q_{k-1}$ to ensure it is less than $q_{k-1}$, and so on.

There are two further steps to the proof. We must first find numbers $m_{k}$ for $k=1, \ldots, n$ such that $a>m_{k} \Rightarrow a \in H_{k}$, and then use the unique normal form to count $I_{k}=\# H_{k} \cap\left[0, m_{k}\right]$ and hence calculate the size of the gap $G_{k}=\mathbb{N}-H_{k}$. Note that the number $m_{k}$ need not be optimal.

Lemma 4.2. $m_{k}=q_{k} m_{k-1}+p_{k}\left(q_{k}-1\right)$.

Lemma 4.3. $I_{k}=q_{k} I_{k-1}+\frac{1}{2}\left(q_{k}-1\right)\left(p_{k}-1\right)$.

We will delay the proofs of these two results. Thus,

$$
\left|G_{k}\right|=m_{k}+1-I_{k}=q_{k}\left|G_{k-1}\right|+\frac{1}{2}\left(q_{k}-1\right)\left(p_{k}-1\right),
$$

and the size of the Weierstrass gap, which is the arithmetic genus given in (3), satisfies the same recursion relation. Thus it is sufficient to prove the theorem for a single Puiseux pair $\left(p_{1}, q_{1}\right)$.

Since $\left(p_{1}, q_{1}\right)=1$, given $1 \leq r \leq q_{1}$ we can choose $1 \leq a<q_{1}$ and $1 \leq b<p_{1}$ such that $a p_{1}-b q_{1}=r$. Then

$$
\begin{aligned}
p_{1} q_{1}+r & =p_{1} q_{1}+a p_{1}-b q_{1} \\
& =\left(p_{1}-b\right) q_{1}+a p_{1} \in H_{1}
\end{aligned}
$$

for each $r=1, \ldots, q_{1}$ and hence for any $r \geq 1$; so $m_{1}=p_{1} q_{1}$. (In fact, the same argument shows that we can use $m_{1}=p_{1} q_{1}-p_{1}-q_{1}$, but that is not necessary here.)

Also, $I_{1}=\#\left\{a_{0} q_{1}+a_{1} p_{1} \leq p_{1} q_{1}\right\}=(1 / 2)\left(p_{1}+1\right)\left(q_{1}+1\right)$ by counting integer lattice points under $q_{1} x+p_{1} y=p_{1} q_{1}$. (The value $p_{1} q_{1}$ is represented twice, and only counted once.)

Hence $\left|G_{1}\right|=p_{1} q_{1}+1-(1 / 2)\left(p_{1}+1\right)\left(q_{1}+1\right)=(1 / 2)\left(p_{1}-1\right)\left(q_{1}-1\right)$, which is the arithmetic genus and hence equals the size of the Weierstrass gap. 
Proof of Lemma 4.2. Since $q_{k} H_{k-1} \subset H_{k}$, then $q_{k}\left(m_{k-1}+1\right) \in H_{k}$. Any $a>$ $p_{k} q_{k}-p_{k}-q_{k}$ lies in the semi-group generated by $p_{k}$ and $q_{k}$; hence any $a>$ $q_{k}\left(m_{k-1}+1\right)+p_{k} q_{k}-p_{k}-q_{k}$ lies in $H_{k}$. Thus $m_{k}=q_{k} m_{k-1}+p_{k}\left(q_{k}-1\right)$.

Proof of Lemma 4.3. Since $m_{k}=q_{k} m_{k-1}+p_{k}\left(q_{k}-1\right)$, and since we may choose $a_{k}<q_{k}$, we get

$$
\begin{aligned}
I_{k} & =\#\left\{\sum_{i=0}^{k} a_{i} \cdot p_{i} q_{i+1} \ldots q_{k} \leq m_{k}\right\} \\
& =\#\left\{\sum_{i=0}^{k-1} a_{i} \cdot p_{i} q_{i+1} \ldots q_{k-1} \cdot q_{k} \leq q_{k} m_{k-1}\right\} \quad\left(\text { when } a_{k}=q_{k}-1\right) \\
& +\#\left\{\sum_{i=0}^{k-1} a_{i} \cdot p_{i} q_{i+1} \ldots q_{k-1} \cdot q_{k} \leq q_{k} m_{k-1}+p_{k}\right\} \quad\left(a_{k}=q_{k}-2\right) \\
& \vdots \\
& +\#\left\{\sum_{i=0}^{k-1} a_{i} \cdot p_{i} q_{i+1} \ldots q_{k-1} \cdot q_{k} \leq q_{k} m_{k-1}+\left(q_{k}-1\right) p_{k}\right\} \quad\left(a_{k}=0\right) \\
& =q_{k} I_{k-1}+\left[\frac{p_{k}}{q_{k}}\right]+\left[\frac{2 p_{k}}{q_{k}}\right]+\ldots+\left[\frac{\left(q_{k}-1\right) p_{k}}{q_{k}}\right] \\
& =q_{k} I_{k-1}+\frac{1}{2}\left(q_{k}-1\right)\left(p_{k}-1\right) .
\end{aligned}
$$

The $i$ th of the $q_{k}$ terms contributes $I_{k-1}$ plus the number of multiples of $q_{k}$ less than $(i-1) p_{k}$, given by $\left[i p_{k} / q_{k}\right]$, the greatest integer part of $i p_{k} / q_{k}$, and hence the second to last expression $q_{k} I_{k-1}+\ldots$ follows. The sum of greatest integer parts is obtained by counting integer lattice points under the graph $p_{k} x=q_{k} y$.

\section{Polynomially parametrised Curves}

If $\Sigma$ is a polynomially parametrised curve, then any deformation within the class of polynomially parametrised curves preserves the irreducible components globally and hence locally. Hence any such deformation that also preserves the link at infinity is an LCP deformation, and we can apply Theorem 1 to show that the Orevkov invariant of $\Sigma$ depends only upon its connected component of polynomially parametrised curves with a given link at infinity.

In this section, we will describe the moduli space of polynomially parametrised curves. This will allow us to study the connected components of the moduli space, and, in particular, when such moduli spaces are connected.

5.1. Moduli spaces. We will begin with a description of the moduli space of irreducible polynomially parametrised curves with a given splice diagram at infinity.

Theorem 5.1. Let the pair of polynomials $(x(t), y(t))$ define a reduced rational curve $\Sigma$. Then the following two properties are equivalent:

(A) The link at infinity of $\Sigma$ has splice diagram

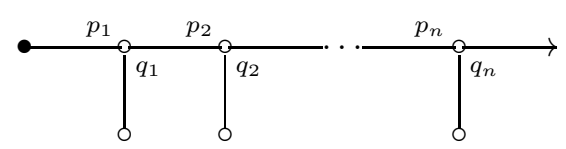


(B) The defining polynomial for $\Sigma$ is given by $P(x, y)=y_{n+1}\left(x, y_{1}, y_{2}, \ldots, y_{n}\right)$, where $y_{1}=y$ and, for each $k=1, \ldots, n$,

$$
y_{k+1}\left(x, y_{1}, y_{2}, \ldots, y_{k}\right)=y_{k}^{q_{k}}+\sum_{e} a_{e} x^{e_{0}} y_{1}^{e_{1}} y_{2}^{e_{2}} \ldots y_{k}^{e_{k}},
$$

where $e=\left(e_{0}, \ldots, e_{k}\right)$ is summed over all tuples such that the order of the pole at $\infty$ (the t-degree) of the corresponding monomial is no greater than the order of the pole at $\infty$ of $y_{k}^{q_{k}}$. As a polynomial in $t, \operatorname{deg}_{t} y_{k}=p_{k} q_{k+1} q_{k+2} \ldots q_{n}$ for $\left(p_{k}, q_{k}\right)=1$ and $y_{n+1} \equiv 0$.

Proof. $(A) \Rightarrow(B)$. Given a curve parametrised by $(x(t), y(t))$, there is an algorithm to determine the polynomial that vanishes on the curve, $P(x, y)=0$. The splice diagram at infinity guides the algorithm, and the holomorphic functions $\left\{y_{2}, \ldots, y_{n}\right\}$ with the stated properties are produced along the way. The algorithm runs as follows.

Take a linear combination $y_{10}=y^{q_{1}}+\lambda x^{p_{1}}$ so that $\operatorname{deg}_{t} y_{10}<p_{1} q_{1}$. By iteratively adding monomials $y_{1, k+1}=y_{1 k}+\alpha_{i j} x^{i} y^{j}$, we can get rid of successively lower powers of $t$, reducing the degree, so that $\operatorname{deg}_{t} y_{1, k+1}<\operatorname{deg}_{t} y_{1 k}$. This procedure stops when we can no longer reduce the degree using monomials in $x$ and $y$, and produces $y_{2}(x, y)$ with pole at infinity of order $\operatorname{deg}_{t} y_{2}=p_{2} q_{3} \ldots q_{n}$ for the following reason.

The order of the pole of $y_{2}(x, y)$ lies in the Weierstrass semi-group $W$ of the curve $\Sigma$. By Theorem 4.1 this is the semi-group generated by the orders of the poles of $x$ and $y$ and the approximate roots. Use the ordering of the approximate roots to define sub-semi-groups

$$
W_{1} \subset W_{2} \subset \ldots \subset W_{n}=W,
$$

where $W_{k}$ is given by those elements of $W$ generated by $q_{1} q_{2} \ldots q_{n}, p_{1} q_{2} \ldots q_{n}$, $p_{2} q_{3} \ldots q_{n}, \ldots, p_{k} q_{k+1} \ldots q_{n}$. In terms of $H_{k}$ defined in the proof of Theorem 4.1 $W_{k}=q_{n} q_{n-1} \ldots q_{k+1} H_{k}$. The polynomial $y_{2}(x, y)$ behaves in a similar way to the approximate root $P_{2}$, in that it meets the splice diagram at infinity below the second Puiseux pair. This is because it meets the splice diagram at infinity before the third Puiseux pair since it possesses only a single Puiseux pair itself; so the order of its pole lies in $W_{2}$, and the order of its pole does not lie in $W_{1}$ since then we could reduce the degree of $y_{2}(x, y)$ further by a polynomial in $x$ and $y$ and we should not have stopped the procedure.

By the semi-group condition of Abhyankar and Moh, $p_{2}$ lies in the semi-group generated by $p_{1}$ and $q_{1}$ or, equivalently, one can reduce the $t$-degree of $y_{2}^{q_{2}}$ by subtracting a monomial in $x$ and $y$. As before, we iteratively reduce the $t$-degree of $y_{2}^{q_{2}}$ using monomials in $x, y$ and $y_{2}$, and produce $y_{3}$ when the procedure stops. The same argument as above shows that $\operatorname{deg}_{t} y_{3}=p_{3} q_{4} \ldots q_{n}$, and the algorithm continues. Finally we are left with $y_{n+1}$, which consists of powers of $t$ that lie in the Weierstrass gap. But then $y_{n+1} \equiv 0$, since no holomorphic functions on $\Sigma$ can have such poles.

Note that the $y_{k}$ 's are not the approximate roots of the defining polynomial of the curve. In fact, the polynomial $y_{k}$ is not well-defined, since there are relations amongst the variables $x, y_{1}, y_{2}, \ldots$ and also since $y_{k}$ can be adjusted by low-degree terms. 
$(B) \Rightarrow(A)$ The expansion of $y$ in terms of $x$ is most easily seen by putting $x=w^{-q_{1} \ldots q_{n}}$; so $w$ is a branch of $x^{-1 / q_{1} \ldots q_{n}}$. This allows us to solve for

$$
t=a_{-1} w^{-1}+a_{0}+a_{1} w+a_{2} w^{2}+\ldots, a_{-1} \neq 0,
$$

and then

$$
y=w^{-p_{1} q_{2} \ldots q_{n}}\left(r_{1}\left(w^{q_{2} \ldots q_{n}}\right)+\cdots+w^{-e_{k} q_{k+1} \ldots q_{n}}\left(r_{k}\left(w^{q_{k+1} \ldots q_{n}}\right)+\ldots,\right.\right.
$$

where $w^{-e_{k} q_{k+1} \ldots q_{n}}$ is the first term with exponent not divisible by $q_{k} q_{k+1} \ldots q_{n}$ and $q_{k+1} \ldots q_{n}$ is defined by requiring that $\left(e_{k}, q_{k}\right)=1$. Note that an expansion for $y$ in terms of $w$ always exists in such a form, but a priori $e_{k} q_{k+1} \ldots q_{n}$ does not necessarily equal $\Delta_{k} q_{k+1} \ldots q_{n}$.

Now suppose there exists $y_{2}(x, y)$ satisfying B1, B2 and B3. Then we can express $y_{2}=w^{-p_{2} q_{3} \ldots q_{n}} \eta(w)$ for $\eta$ holomorphic and $\eta(0) \neq 0$. The leading (most negative) power of $w$ is the same as the leading power of $w$ not divisible by $q_{2} \ldots q_{n}$. Hence it comes from the term $y^{q_{1}}$. Thus

$$
-p_{2} q_{3} \ldots q_{n}=-q_{1} p_{1} q_{2} q_{3} \ldots q_{n}-e_{2} q_{3} \ldots q_{n} .
$$

Any other term $x^{i} y^{j}$ does not contribute, because it gives a more positive power, since at best one can get

$$
i q_{1} \ldots q_{n}+j p_{1} \ldots q_{n}-e_{2} q_{3} \ldots q_{n}<q_{1} p_{1} \ldots q_{n}-e_{2} q_{3} \ldots q_{n}
$$

since $i q_{1}+j p_{1}<p_{1} q_{1}$.

More generally, $y_{k}=w^{-p_{k} q_{k+1} \ldots q_{n}} \eta(w)$ for $\eta$ holomorphic and $\eta(0) \neq 0$. The leading power of $w$ not divisible by $q_{k} q_{k+1} \ldots q_{n}$ comes from the term $y_{k-1}^{q_{k-1}}$; so

$$
-p_{k} q_{k+1} \ldots q_{n}=q_{k-1} p_{k-1} q_{k} \ldots q_{n}-e_{k} q_{k+1} \ldots q_{n}
$$

and no other term $y_{k-1}^{i_{k-1}} \ldots y_{1}^{i_{1}} x^{i_{0}}$ contributes, since it can at best give the weighted degree of the monomial minus $e_{k} q_{k} \ldots q_{n}$, and the weighted degree of the monomial is strictly less than $q_{k-1} p_{k-1} q_{k} \ldots q_{n}$.

From (6) we see that $e_{k}=\Delta_{k}=p_{k}-p_{k-1} q_{k-1} q_{k}<0$ by (1) ii.

Here is an example to demonstrate the algorithm.

Example. Let $x(t)=t^{12}+t$ and $y(t)=t^{8}+t^{2}$. The algorithm of the previous proof enables us to produce the defining polynomial of $x$ and $y$ and to prove that its splice diagram at infinity is

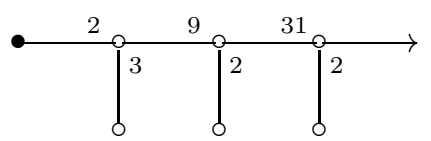

Step 1. $\operatorname{deg}_{t}\left\{x^{2}-y^{3}\right\}=18$; so set $y_{2}(t)=x^{2}-y^{3}$;

Step 2. $\operatorname{deg}_{t}\left\{\left(x^{2}-y^{3}\right)^{2}-9 x^{3}\right\}=31$; so set $y_{3}(t)=\left(x^{2}-y^{3}\right)^{2}-9 x^{3}$;

Step 3. $\operatorname{deg}_{t}\left\{\left(\left(x^{2}-y^{3}\right)^{2}-9 x^{3}\right)^{2}+(16 / 3) y \cdot y_{2}^{3}\right\}=61$;

Step 4 . add to step 3 a multiple of $x \cdot y_{2} \cdot y_{3}$ to get rid of the $t^{61}$ term;

Step 5. continue this to get rid of $t^{60}, t^{59}, \ldots, t^{0}$ and find that only powers of $t$ that lie in the semi-group generated by $12,8,18$ and 31 arise;

Step 6. thus, $P(x, y)=\left(\left(x^{2}-y^{3}\right)^{2}-9 x^{3}\right)^{2}+(16 / 3) y\left(x^{2}-y^{3}\right)^{3}+\ldots$. Alternatively, in step $2, \operatorname{deg}_{t}\left\{\left(x^{2}-y^{3}\right)^{2}-9 x y^{3}\right\}=31$; so we may set $y_{3}(t)=$ $\left(x^{2}-y^{3}\right)^{2}-9 x y^{3}$ and continue with the algorithm. Although the process is not unique, and hence $P$ is not unique as a polynomial in $x, y, y_{2}$ and $y_{3}$, as a polynomial in $x, y, P(x, y)$ is unique. 
The example demonstrates both the working of the algorithm and the fact that the $y_{j}$ are not unique, in contrast with the unique approximate roots.

The conditions (B) in Theorem 5.1 lead to an explicit expression for the moduli space of polynomially parametrised curves with given splice diagram at infinity as an algebraic variety. For $x(t)=\sum a_{i} t^{i}$ and $y=\sum b_{j} t^{j}$, the variety is given as a set of polynomial equations in the coefficients $\left\{a_{i}, b_{j}\right\}$ as follows.

The expression for $y_{k+1}$ in (4) a priori has $t$-degree $p_{k} q_{k} q_{k+1} \ldots q_{n}$, but since we require that $y_{k+1}$ has $t$-degree $p_{k+1} q_{k+2} q_{k+3} \ldots q_{n}<p_{k} q_{k} q_{k+1} \ldots q_{n}$, by (1) ii, then each of the coefficients of $t^{m}$ for $p_{k} q_{k} q_{k+1} \ldots q_{n} \geq m>p_{k+1} q_{k+2} q_{k+3} \ldots q_{n}$ must vanish. For $m \in W_{k}$, defined in (5), the coefficient of $t^{m}$ is canceled by a monomial in $x$ and $y_{i}, i \leq k$. For each $m \notin W_{k}$, define $T_{m, k}\left(\left\{a_{i}, b_{j}\right\}\right)$ to be the coefficient of $t^{m}$ in $y_{k}$. We call the variety $\left\{T_{m, k}=0\right\}$ the moduli space of polynomially parametrised curves with given splice diagram at infinity.

Each polynomial $T_{m, k}$ has the property that it only depends on $b_{j}$ and $a_{i}$ for $i \geq e(k)-m$, where $e(1)=p_{1} q_{1} q_{2} \ldots q_{n}$ and $e(k)=e(k-1)+\left(q_{k}-1\right) p_{k} q_{k+1} \ldots q_{n}$, and if equality occurs, then that coefficient appears in $T_{m, k}$ linearly with nonzero coefficient. By nonzero coefficient, we mean that it is a polynomial in the $\left\{b_{j}\right\}$ that is required to be nonzero (earlier in the algorithm).

When the link at infinity has more than one component or, equivalently, the polynomially parametrised curves are reducible, if the splice components meet only on the root vertex or, equivalently, the curve components do not intersect at infinity, then the moduli space is simply a product of the moduli spaces for each component, minus a divisor along which the curves meet at infinity. In general, any two irreducible components of the link at infinity agree along an initial iterated cabling, and this introduces a further equation giving equality of the initial coefficients of the respective pairs of polynomials.

It is often difficult to tell when the variety is connected or even when it is nonempty. In the case of a knot at infinity, the semi-group theorem of Abhyankar and Moh [1] and its converse by Sathaye and Stenerson [19] give necessary and sufficient conditions for the moduli space of curves (not necessarily rational) to be nonempty.

5.2. Solving the equations. In the previous section, we constructed a variety that gives the moduli space of an irreducible component of a polynomially parametrised curve with a given link at infinity. In this section, we will be more explicit, and solve the equations for a given link at infinity. It is important to note that we only consider splice diagrams that are links at infinity of algebraic curves; so $\Delta_{k}=$ $p_{k}-p_{k-1} q_{k-1} q_{k}<0$ for all $k$.

We will begin with a short discussion of the canonical class divisor associated to $L_{\Sigma}$. The splice diagram for $L_{\Sigma}$ gives an efficient resolution at infinity for $\Sigma$. It gives the valency $>2$ curves in the plumbing diagram of the divisor at infinity $D \subset X_{\Sigma}$. It can be arranged that the canonical class $K$ of $X_{\Sigma}$ is supported on the divisor $D$; so $K$ is given by a sequence of multiplicities, one for each irreducible curve in $D$. At each component of $L_{\Sigma}$, the multiplicity of $K$ is given by the local intersection $K \cdot \Sigma$ at that component. Instead of working with $K \cdot \Sigma$, we prefer to work with $-K \cdot \Sigma-1$, which we denote by $d$ at each component of the link, and more generally at each virtual component of the link, or node of the splice diagram.

The number $d$ can be calculated at a node in the splice diagram of $L_{\Sigma}$ by taking a path from the node to the root- the node that represents the proper transform 


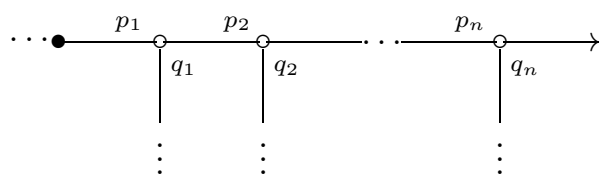

Figure 2. Path in splice diagram at infinity

of $\mathbb{P}^{1}=\mathbb{P}^{2}-\mathbb{C}^{2}$ - of the splice diagram. Such a path is represented by Figure 2 where other arrows may exist in the diagram at the places marked with dots. If we notate $d$ at the $k$ th node by $d_{k}$, then $d_{k}$ is calculated recursively by

$$
d_{k}=q_{k} d_{k-1}+\Delta_{k}, \quad d_{1}=p_{1}+q_{1} .
$$

The following theorem is a more precise statement of the "sufficiently negative" condition of Theorem 2 ,

Theorem 5.2. When $d_{n} \geq p_{1} q_{2} \ldots q_{n}-1$ for each component of $L$, the moduli space of polynomially parametrised curves $\Sigma$ with $L_{\Sigma}=L$ is connected.

Proof. To start, consider each irreducible component of $\Sigma$ separately, and hence use the description of the moduli space given in the previous section. A straightforward way to solve the equations is to arbitrarily choose the coefficients of $y(t)$, and solve for the coefficients of $x(t)$, and use this to show that when the moduli space is nonempty, it is connected. As described in the properties of the $T_{m, k}$, the equations $T_{m, k}=0$ form a diagonal system in which each coefficient of $x$ appears for the first time linearly with nonzero coefficient.

For each $k=2, \ldots, n$, the equation $\operatorname{deg}_{t} y_{k}=p_{k} q_{k+1} q_{k+2} \ldots q_{n}$ gives rise to

$$
p_{k-1} q_{k-1} q_{k} q_{k+1} q_{k+2} \ldots q_{n}-1-p_{k} q_{k+1} q_{k+2} \ldots q_{n}=-\Delta_{k} q_{k+1} q_{k+2} \ldots q_{n}-1
$$

vanishing coefficients, since a priori the degree of $y_{k}$ is $p_{k-1} q_{k-1} q_{k} q_{k+1} q_{k+2} \ldots q_{n}-1$. The coefficients of powers of $t$ that do not lie in the sub-semi-group $W_{k}$ give rise to the equations $T_{m, k}=0$, and these are easily solved, as a diagonal system when the number of coefficients of $x$ is no less than the number of equations. (In fact, many coefficients of $x$ are wasted, since we need only solve $T_{m, k}=0$ for $m \notin W_{k}$.) We need

$$
q_{1} q_{2} \ldots q_{n} \geq \sum_{k=2}^{n} \Delta_{k} q_{k+1} q_{k+2} \ldots q_{n}+1
$$

Put

$$
a(n)=\left(q_{1}+p_{1}\right) q_{2} \ldots q_{n}+\sum_{k=2}^{n} \Delta_{k} q_{k+1} q_{k+2} \ldots q_{n}-d_{n}
$$

so (9) becomes

$$
d_{n} \geq p_{1} q_{2} \ldots q_{n}-1-a(n) .
$$

Using $\Delta_{n}-d_{n}=-d_{n-1} q_{n}$, we see that $a(n)=a(n-1) q_{n}$ or $a(k)=a(k-1) q_{k}$ for all $k$; and since $a(2)=0$, by induction $a(n)=0$. Hence, $d_{n} \geq p_{1} q_{2} \ldots q_{n}-1$.

The proof is not complete, since the vanishing of the specified coefficients only guarantees $\operatorname{deg}_{t} y_{k} \leq p_{k} q_{k+1} \ldots q_{n}$ and not $\operatorname{deg}_{t} y_{k}=p_{k} q_{k+1} \ldots q_{n}$. If, by good fortune, we do get equality each time, then we can calculate the dimension of the 
space of solutions to the system as the degree of $y(t)$ plus the number of coefficients of $x(t)$ unused when solving the system.

Lemma 5.3. The moduli space of polynomially parametrised curves with given link at infinity is either of the expected dimension and connected, or empty.

Proof. We have arranged that the coefficient of $t^{p_{k} q_{k+1} q_{k+2} \ldots q_{n}+1}$ vanishes. The coefficient of $t^{p_{k} q_{k+1} q_{k+2} \ldots q_{n}}$ is a polynomial $B_{k}$ in the coefficients of $y(t)$. One of two cases must occur. Either

(i) the polynomial $B_{k}$ is identically zero, and thus the moduli space of polynomially parametrised curves with that particular link at infinity is empty; or

(ii) the polynomial $B_{k}$ is not identically zero, and thus the coefficients of $y(t)$ that give a smaller degree for $y_{k}$ form a set of codimension $\geq 1$ and the moduli space of coefficients of $y(t)$ that give the right degree for $y_{k}$ is a nonempty connected space.

If each component of $L_{\Sigma}$ gives rise to a nonempty moduli space of polynomially parametrised curves, then by construction we can fit the moduli spaces of the components of $\Sigma$ together to get the full moduli space. Two link components agree along an initial path of cablings or, equivalently, the respective compactification divisors at infinity agree on an initial path of blow-ups, when the same top coefficients of $y(t)$ are chosen along with the same polynomials $y_{k}$. Again, either accidental agreement along a further path of cablings occurs on a codimension $\geq 1$ set, or the moduli space of polynomially parametrised curves with given link at infinity is empty.

This completes the proof of the theorem.

Remarks. (i) We can ensure that each nonempty moduli space contains an immersion. When an unused coefficient $a_{i}$ of $x$ appears for the first time, and hence linearly, in $T_{m, k}$, say, we can use $a_{i}$ as a parameter. The polynomial $x(t)$ then depends on the parameter $a_{i}$, and $\left(x^{\prime}(t), y^{\prime}(t)\right) \neq(0,0)$ for a generic choice of $a_{i}$.

(ii) In the next section, we prove that for positive braids, Lemma 5.3 can be strengthened to show that the space of solutions is always nonempty.

We are now almost in a position to prove Theorem [2. The following lemma proves that a particular class of immersions has abelian fundamental group and hence abelian Orevkov invariant.

Lemma 5.4. Let $\Sigma=\sqcup S_{i} \rightarrow \mathbb{C}^{2}$ be an immersion. If $2 g\left(\Sigma_{i}\right)-2>K \cdot \bar{\Sigma}_{i}$ on each component $\Sigma_{i}$, then $\pi_{1}\left(\mathbb{C}^{2}-\Sigma\right)$ is abelian.

Proof. This is a simple application of Nori's theorem. First, assume that the immersion of $\Sigma$ is nodal. Then, on each component,

$$
\bar{\Sigma}_{i} \cdot \bar{\Sigma}_{i}+2-2 p_{a}\left(\Sigma_{i}\right)=-K \cdot \bar{\Sigma}_{i},
$$

and since the arithmetic genus is given by $p_{a}\left(\Sigma_{i}\right)=g\left(\Sigma_{i}\right)+r\left(\Sigma_{i}\right)$, where $r\left(\Sigma_{i}\right)$ is the number of double points of $\Sigma_{i}$, then we have

$$
\bar{\Sigma}_{i} \cdot \bar{\Sigma}_{i}-2 r\left(\Sigma_{i}\right)=-K \cdot \bar{\Sigma}_{i}+2 g-2,
$$

and when this is strictly positive, for each component $\Sigma_{i}$, Nori's theorem applies to let us conclude that $\pi_{1}\left(\mathbb{C}^{2}-\Sigma\right)$ is abelian.

When the immersion of $\Sigma$ is not nodal, blow up triple points and higher multiplicity points. Add to the divisor at infinity the exceptional curves whose union is 
given by $E$, so that Nori's theorem can be applied to $\tilde{S} i \rightarrow X-D \cup E$. Then after each blow-up, the number $\bar{\Sigma}_{i} \cdot \bar{\Sigma}_{i}$ (use $\Sigma$ to denote its proper transform also) is reduced by the same amount as the number $-K \cdot \bar{\Sigma}_{i}$, which maintains the inequality, and the arithmetic genus is possibly reduced, which improves the inequality. Thus, the general case follows from the nodal case.

Proof of Theorem Q By the invariance of $\mathcal{O}(\Sigma)$ under LCP deformations and the connectedness of the moduli space of polynomially parametrised curves under LCP deformations, it is sufficient to calculate $\mathcal{O}(\Sigma)$ for one curve $\Sigma$ with given link at infinity.

As explained in Remark (ii) after Theorem 5.2, each moduli space contains an immersion. When each component is rational with one point at infinity, the condition $2 g\left(\Sigma_{i}\right)-2>K \cdot \bar{\Sigma}_{i}$ in Lemma 5.4 becomes $-K \cdot \bar{\Sigma}_{i}-1>1$, and the left-hand side is the number $d$ we associate to the link component of the splice diagram. The condition $d>1$ is certainly fulfilled on each component, since $d_{n} \geq p_{1} q_{2} \ldots q_{n}-1>1$. Hence $\mathcal{O}(\Sigma)$ is abelian and this is true on the entire moduli space; so the theorem is proven.

5.3. Positive braids. The link at infinity of an affine algebraic curve inherits a natural braid structure. In fact, there is a braid structure for each line in $\mathbb{C}^{2}$, obtained by projection along the line. The condition that the splice diagram of a link at infinity gives a positive braid is a condition on the weights $p_{i}, q_{i}$ of each branch of the splice diagram given in Figure 2,

In order to make the largest possible set of links at infinity positive braids, we choose to project along the $x$ direction (assume $\operatorname{deg} x(t)>\operatorname{deg} y(t)$ ). In the following proposition, if we were to use the braid structure from the projection along the $y$ direction, then the condition $d_{n}>p_{1} q_{2} \ldots q_{n}$ would be replaced by $d_{n}>q_{1} q_{2} \ldots q_{n}>p_{1} q_{2} \ldots q_{n}$, which is a stronger condition, and hence fewer links would be positive braids.

Proposition 5.5. The link at infinity of an affine curve is a positive braid precisely when $d_{n}>p_{1} q_{2} \ldots q_{n}$ along each path of the splice diagram shown in Figure?

Proof. Each component of a positive braid is again a positive braid; so we will restrict to each path in the splice diagram. We will actually prove that $d_{k}>$ $p_{1} q_{2} \ldots q_{k}, k=1, \ldots, n$. Thus we claim that the corresponding virtual link components are also positive braids.

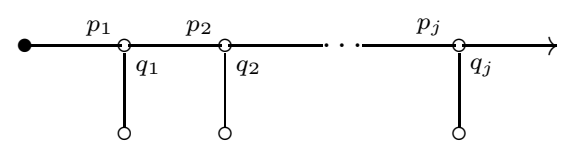

FiguRE 3.

Lemma 5.6. Using the framing supplied by the braid projection, the self-linking number of the braid in Figure 3 is

$$
l_{j}=p_{j} q_{j}+p_{1} q_{2} \ldots q_{j}-d_{j} .
$$

Equivalently, it is the braid index of the braid. 
Proof. We prove this by induction. To begin, the $\left(p_{1}, q_{1}\right)$ torus knot has selflinking number $l_{1}=p_{1} q_{1}-q_{1}=p_{1} q_{1}+p_{1}-d_{1}$, as required. To see the recursive relationship for $l_{j}$, suppose we cable $q_{j+1}$ parallel strings along Figure 3 using the framing supplied by the braid projection. Then the self-linking number, or braid index, of this new braid is given by $q_{j+1}^{2} l_{j}$. Now consider a $q_{j+1}$ cabling on Figure 3 with weight $p_{j+1}$. Note that $p_{j+1}$ gives the linking number of (the virtual copy of) Figure 3 with each of the $q_{j+1}$ cables, and a cabling already links $q_{j+1} l_{j}$ times with (the virtual copy of) Figure 3 Thus, the new self-linking number is

$$
l_{j+1}=q_{j+1}^{2} l_{j}+\left(q_{j+1}-1\right)\left(p_{j+1}-q_{j+1} l_{j}\right)=q_{j+1} l_{j}+q_{j+1} p_{j+1}-p_{j+1} .
$$

In particular, $l_{j}-p_{j} q_{j}$ and $p_{1} q_{2} \ldots q_{j}-d_{j}$ satisfy the same recursion relation. Since $l_{1}-p_{1} q_{1}=p_{1}-d_{1}$, the result follows.

Now, a positive braid arises when the number $p_{j+1}-q_{j+1} l_{j}$ of extra twists in (10) is positive. Hence $p_{k}>l_{k-1} q_{k}, k=1, \ldots, n$, and $d_{k}>p_{1} q_{2} \ldots q_{k}$ for $k=1, \ldots, n$.

Note, too, that the recursive relationship for $d_{j}$ shows that $d_{n}>p_{1} q_{2} \ldots q_{n}$ implies $d_{k}>p_{1} q_{2} \ldots q_{k}$ for $k=1, \ldots, n$.

Since the condition in Proposition 5.5 is stronger than that in Theorem 5.2 , we get the following corollary.

Corollary 5.7. The moduli space of polynomially parametrised curves with positive braid at infinity is connected.

Proposition 5.8. The moduli space of polynomially parametrised curves with positive braid at infinity is nonempty.

Proof. In the proof of Theorem 5.2 we produced points in the variety for a given splice diagram by solving for the coefficients of $f$ in terms of the coefficients in $g$. The variety can be empty when there is accidental vanishing of the coefficients of any of $t^{p_{k} q_{k+1} \cdots q_{n}}$ for $k=1, \ldots, n$ (where $n$ is the number of Puiseux pairs of the particular component). Since at each component of a positive braid there are $n-1$ extra degrees of freedom,

$$
d_{n} \geq p_{1} q_{2} \ldots q_{n}+1 \geq p_{1} q_{2} \ldots q_{n}-1,
$$

then these can be used to set the coefficients of $t^{p_{k} q_{k+1} \cdots q_{n}}$ equal to 1 . Thus, for each component, the moduli space is nonempty.

When we put the components together, components branching off at different points in the splice diagram correspond to different choices of coefficients that lie in the semi-group of the curve, and hence waste no degrees of freedom. Thus the moduli space of polynomial curves with positive braid at infinity is nonempty.

For positive braids, we can prove Theorem 2 without using Nori's theorem. We used Nori's theorem to calculate the Orevkov invariant for a special representative in the moduli space of polynomially parametrised curves. For positive braids, we can do such a calculation explicitly.

Theorem 5.9. Given a curve $(F(t), G(t))$, define

$$
(f(t), g(t))=\left(F\left(t^{d}\right)+\epsilon h(t), G\left(t^{d}\right)\right) .
$$

Then for almost all $h$ of degree $<d$ and for small enough $\epsilon$, the Orevkov invariant of $(f(t), g(t))$ is a quotient of the Orevkov invariant of $(F(t), G(t))$. 
Proof. Choose a large ball $B_{R}$ that realises the link at infinity of $(F(t), G(t))$. Choose a path from 0 to $\infty$ in $\mathbb{C}^{*}$ that avoids self-intersections of $(F, G)$. Choose $\epsilon$ small enough so that a tube of radius $\epsilon R$ intersects the curve $\left(F\left(t^{d}\right), G\left(t^{d}\right)\right)$ only in an embedded strip in the curve. Thus, along the path, the $d$ deformations remain disjoint from the rest of $(F, G)$. The $d$ deformations may intersect each other. The branch point at $t=0$ gives the relation that each of the $d$ elements in the fundamental group are equal. Thus, the Orevkov invariant of the deformed curve $(f(t), g(t))=\left(F\left(t^{d}\right)+\epsilon t, G\left(t^{d}\right)\right)$ is a quotient of the Orevkov invariant of $(F(t), G(t))$. The Orevkov invariants are the same if $B_{R}$ realises the link at infinity of $(f(t), g(t))$.

Next we give a construction of a rational curve with positive braid at infinity in order to apply Theorem 5.9 .

Theorem 5.10. If $(F(t), G(t))$ defines the component of a positive braid

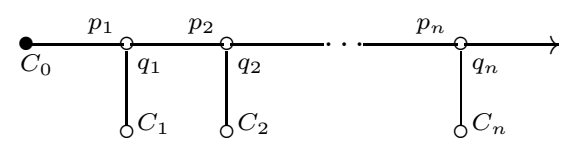

then for $\operatorname{deg} h(t)<\left(d_{n}-q_{1} q_{2} \ldots q_{n}\right) q_{n+1}$, the curve

$$
(f(t), g(t))=\left(F\left(t^{q_{n+1}}\right)+\epsilon h(t), G\left(t^{q_{n+1}}\right)\right)
$$

defines the component

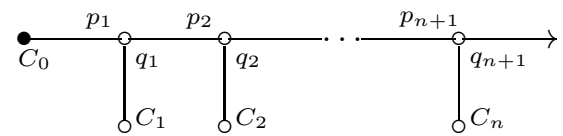

where any $p_{n+1}$ that satisfies $p_{n+1}-p_{n} q_{n} q_{n+1}+d_{n} q_{n+1}=d_{n+1}>p_{1} q_{2} \ldots q_{n} q_{n+1}$ is uniquely determined by the choice of $h$.

Proof. When we solve the diagonal system of equations $T_{m, k}=0$ for a positive braid at infinity to get $(F(t), G(t))$, the coefficients of $F(t)$ appear in order from the highest power of $t$ to the lowest. Furthermore, the last $d_{n}-q_{1} q_{2} \ldots q_{n}$ coefficients of $F(t)$ are irrelevant to the system of equations. If the degree of $\epsilon h(t)$ is less than $\left(d_{n}-q_{1} q_{2} \ldots q_{n}\right) q_{n+1}$, then $\epsilon h(t)$ does not affect the equations $T_{m, k}=0$ for $k<n$, and features only in the equations $T_{m, n}=0$. The equations $T_{m, n}=0$ form a diagonal system in the coefficients of $\epsilon h(t)$, and hence we can solve for any $p_{n+1}$ that gives rise to a positive braid or, equivalently, satisfies the inequality $p_{n+1}-p_{n} q_{n} q_{n+1}+d_{n} q_{n+1}=d_{n+1}>p_{1} q_{2} \ldots q_{n} q_{n+1}$.

Thus, by induction, the Orevkov invariant of a polynomially parametrised curve $\Sigma$ with positive braid at infinity is abelian, since it is a quotient of the Orevkov invariant of a simpler curve obtained by reducing by one the number of Puiseux pairs on a component of $\Sigma$. Eventually the curve is reduced to a configuration of lines, where the invariant is abelian.

\section{Further EXAmples}

The invariance of the Orevkov invariant under LCP deformations reduces the problem of calculating the invariant, or searching for a curve with nonabelian invariant, to understanding the finitely many topological types at infinity of curves with given degree. One can enumerate polynomially parametrised curves by their 
splice diagrams at infinity and the connected components of the moduli space of polynomially parametrised curves with that splice diagram at infinity. When the curves are irreducible, the list for degree up to 12 is as follows:

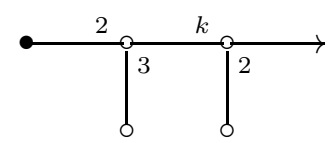

Figure 4. degree $6, k=3,5, \ldots, 11$

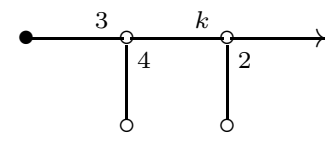

Figure 5. degree $8, k=3,7,9, \ldots, 23$

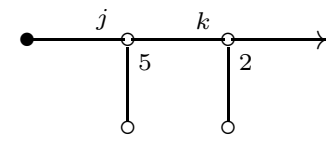

Figure 6. degree $10, j=2,3,4$, odd $k=j m+5 n<10 j$

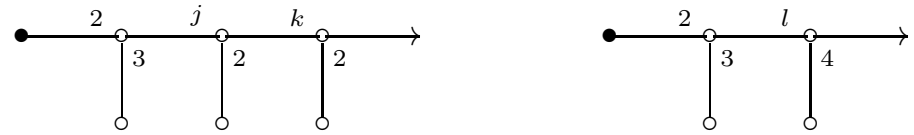

Figure 7 . degree $12, j=3,5, \ldots, 11, k=j, \ldots, 4 j-1, l=3,5, \ldots, 23$
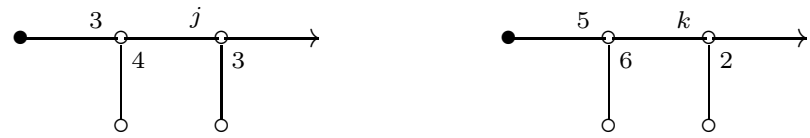

FiguRE 8. degree $12, j=3,7,9, \ldots, 35, k=5,11,15,17,21,23, \ldots, 59$

In each of the diagrams, by $a, \ldots, b$ we mean all of the odd integers from $a$ to $b$, except in the case of $k$ in Figure 7 , which misses some odd values.

The degree $<6,7$ and 11 curves have positive braids at infinity, and we have not listed them, nor positive braids for degree 6, 8, 10 and 12. They are covered by Theorem 2 along with many on the list above (for example, when the degree is 6 , only $k=3$ is not covered). Using MAPLE for those curves above of degree $<12$ not covered by Theorem 2 we have shown that each moduli space of polynomially parametrised curves is connected and that the Orevkov invariant of each is abelian. 
So far we have only been able to calculate the Orevkov invariant of a curve by calculating the fundamental group of the complement of some curve obtained by deforming the original curve and applying Theorem 1 Given the many cases where the Orevkov invariant depends only on the link at infinity, we would hope to be able to calculate the invariant without calculating the fundamental group of the complement of a curve.

One can construct smooth examples of curves $\mathbb{C} \rightarrow \mathbb{C}^{2}$ with nonabelian Orevkov invariant. An example is as follows. Consider the representation of

$$
\pi_{1}(\mathbb{C}-2 \text { points })=\left\{\gamma_{1}, \gamma_{2}\right\}
$$

into the braid group

$$
B_{3}=\left\{\sigma_{1}, \sigma_{2} \mid \sigma_{1} \sigma_{2} \sigma_{1}=\sigma_{2} \sigma_{1} \sigma_{2}\right\}
$$

given by

$$
\gamma_{1} \mapsto \sigma_{2}^{-2} \sigma_{1} \sigma_{2}^{2} \quad \text { and } \quad \gamma_{2} \mapsto \sigma_{1}^{2} \sigma_{2} \sigma_{1}^{-2}
$$

This gives rise to a smoothly embedded surface in $\mathbb{C}^{2}$ described via two maps from a disk $\mathbb{C}$ to $\mathbb{C}$. The first map, $x$, is a 3 -fold branched cover with two branch points. Take a regular point $c$ for $x$, and a loop $\gamma$ starting and ending at $c$ that moves around one of the branch points of $x$. The pre-image $x^{-1}(\gamma)$ is a path containing the three pre-images $x^{-1}(c)$. The second map, $y$, is a 2 -fold branched cover that wraps $x^{-1}(\gamma)$ around its single branch point in such a way that it has a double point.

The map $(x, y): \mathbb{C} \rightarrow \mathbb{C}^{2}$ defines a smoothly embedded surface $\Sigma$ with

$$
\pi_{1}\left(\mathbb{C}^{2}-\Sigma\right)=\{a, b \mid a b a=b a b\} .
$$

This cannot be an algebraic curve, since by the Abhyankar-Moh-Suzuki theorem, any embedded disk is equivalent to the standard disk. We can add double points to this curve, whilst preserving the Orevkov invariant, to make it seem more like a polynomially parametrised curve. Nevertheless, the link at infinity of this does not arise as the link at infinity of an algebraic affine plane curve. It would be interesting to characterise those links at infinity that do arise from a similar construction of a nonabelian Orevkov invariant.

\section{ACKNOWLEDGEMENTS}

The authors would like to acknowledge useful discussions with S. Yu. Orevkov and Gavin Brown.

\section{REFERENCES}

1. S. Abhyankar, On the semigroup of a meromorphic curve, Proc. Internat. Sympos. Algebraic Geometry, Kyoto (1977), 249-414. MR 83h:14020

2. S. A. Broughton, On the topology of polynomial hypersurfaces, Proc. Amer. Math. Soc. Sympos. Pure Math. 40 (1983), 167-178. MR 85d:14033

3. Pierre Deligne, Le groupe du complement d'une courbe plane n'ayant que des points ordinaires est abelien (d'apres W. Fulton), Seminaire Bourbaki, Lecture Notes in Math. 842, Springer Verlag (1981), 1-10. MR 83f:14026

4. A. Dimca and A. Nemethi, On the monodromy of complex polynomials, Duke Math. J. 108 (2001), 199-209.

5. D. Eisenbud and W. D. Neumann, Three-dimensional link theory and invariants of plane curve singularities, Ann. Math. Stud. 110, Princeton Univ. Press (1985). MR 87g:57007

6. W. Fulton, On the fundamental group of the complement of a node curve, Ann. of Math. 111 (1980), 407-409. MR 82e:14035 
7. S. Kaliman, Rational polynomials with a $\mathbb{C}^{*}$-fiber, Pacific J. Math. 174 (1996), 141-194. MR 97h:14026

8. A. Libgober, Alexander polynomial of plane algebraic curves and cyclic multiple planes, Duke Math. J. 49 (1982), 833-851. MR 84g:14030

9. W. D. Neumann, Complex algebraic curves via their links at infinity, Invent. Math. 3 (1989), 445-489. MR 91c:57014

10. W. D. Neumann, Irregular links at infinity of complex affine plane curves, Quarterly J. Math. 50 (1999), 301-320. MR 2001i:32047

11. W. D. Neumann and P. Norbury, Vanishing cycles and monodromy of complex polynomials, Duke Math. J. 101 (2000), 487-497. MR 2002d:32048

12. W. D. Neumann and P. Norbury, Unfolding polynomial maps at infinity, Math. Ann. 318 (2000), 149-180. MR 2001j:32028

13. W. D. Neumann and L. Rudolph, Unfoldings in knot theory, Math. Ann. 278 (1987), 409-439 and Corrigendum 282 (1988), 349-351. MR 89j:57017a,b

14. Madhav V. Nori, Zariski's conjecture and related problems, Ann. Sci. École Norm. Sup. (4) 16 (1983), 305-344. MR 86d:14027

15. Mutsuo Oka, Two transforms of plane curves and their fundamental groups, J. Math. Sci. Univ. Tokyo 3 (1996), 399-433. MR 97j:14030

16. S. Yu. Orevkov, The fundamental group of the complement of a plane algebraic curve, Mat. Sb. 137 (179) (1988), 260-270; English transl., Math. USSR Sb. 65 (1990), 267-277. MR 90e: 14028

17. S. Yu. Orevkov, The commutant of the fundamental group of the complement of a plane algebraic curve, Russian Math. Surveys 45 (1990), 221-222. MR 91g:14020

18. P. Russell, Good and bad field generators, J. Math. Kyoto Univ. 17 (1977), 319-331. MR 56:2977

19. A. Sathaye and J. Stenerson, On plane polynomial curves, Algebraic geometry and its applications, C.L. Bajaj, Ed., Springer (1994), 121-142. MR 95a:14032

20. J. P. Serre, Algebraic groups and class fields, Graduate Texts in Math. 117, Springer-Verlag (1988). MR 88i:14041

Department of Mathematics, Barnard College, Columbia University, New York, NEW YORK 10027

E-mail address: neumann@math.columbia.edu

Department of Pure Mathematics, Adelaide University, Adelaide, Australia 5005

E-mail address: pnorbury@maths.adelaide.edu.au

Current address: Department of Mathematics, Melbourne University, Parkville, Australia, 3052 